ARTICLE

Received 26 Aug 2016 | Accepted 27 Jan 2017 | Published 17 Mar 2017

DOI: $10.1038 /$ ncomms14766

OPEN

\title{
Mutual reinforcement between telomere capping and canonical Wnt signalling in the intestinal stem cell niche
}

Ting-Lin B. Yang ${ }^{1,2}$, Qijun Chen ${ }^{1}$, Jennifer T. Deng ${ }^{1}$, Geetha Jagannathann', John W. Tobias ${ }^{3}$, David C. Schultz ${ }^{4}$, Shan Wang ${ }^{5}$, Christopher J. Lengner ${ }^{5}$, Anil K. Rustgi ${ }^{6}$, John P. Lynch ${ }^{6}$ \& F. Brad Johnson²,

Critical telomere shortening (for example, secondary to partial telomerase deficiency in the rare disease dyskeratosis congenita) causes tissue pathology, but underlying mechanisms are not fully understood. Mice lacking telomerase (for example, $m T R^{-/}-$telomerase RNA template mutants) provide a model for investigating pathogenesis. In such mice, after several generations of telomerase deficiency telomeres shorten to the point of uncapping, causing defects most pronounced in high-turnover tissues including intestinal epithelium. Here we show that late-generation $m T^{-/-}$mutants experience marked downregulation of Wnt pathway genes in intestinal crypt epithelia, including crypt base columnar stem cells and Paneth cells, and in underlying stroma. The importance of these changes was revealed by rescue of crypt apoptosis and Wnt pathway gene expression upon treatment with Wnt pathway agonists. Rescue was associated with reduced telomere-dysfunction-induced foci and anaphase bridges, indicating improved telomere capping. Thus a mutually reinforcing feedback loop exists between telomere capping and Wnt signalling, and telomere capping can be impacted by extracellular cues in a fashion independent of telomerase.

\footnotetext{
${ }^{1}$ Department of Pathology and Laboratory Medicine, Perelman School of Medicine, University of Pennsylvania, Philadelphia, Pennsylvania 19104, USA.

${ }^{2}$ Cell and Molecular Biology Program, Biomedical Graduate Studies, University of Pennsylvania, Philadelphia, Pennsylvania 19104, USA. ${ }^{3}$ Penn Molecular Profiling Center, Perelman School of Medicine, University of Pennsylvania, Philadelphia, Pennsylvania 19104, USA. ${ }^{4}$ Wistar Institute, University of Pennsylvania, Philadelphia, Pennsylvania 19104, USA. ${ }^{5}$ Department of Animal Biology, School of Veterinary Medicine, University of Pennsylvania, Philadelphia, Pennsylvania 19104, USA. ${ }^{6}$ Division of Gastroenterology, Department of Medicine, Abramson Cancer Center, Perelman School of Medicine, University of Pennsylvania, Philadelphia, Pennsylvania 19104, USA. ${ }^{7}$ Institute on Aging, University of Pennsylvania, Philadelphia, Pennsylvania 19104, USA. Correspondence and requests for materials should be addressed to F.B.J. (email: johnsonb@mail.med.upenn.edu).
} 
$\mathrm{F}$ undamental to intestinal epithelial homeostasis are intestinal stem cells (ISCs), including crypt base columnar (CBC) stem cells and quiescent ' +4 ' ISCs. CBCs divide every few days and are positioned at the crypt base between Paneth cells, which together with underlying stroma, contribute to the $\mathrm{CBC}$ niche by providing intercellular Wnt signals ${ }^{1}$. Canonical Wnt proteins mediate short-range intercellular communication leading within target cells to the inhibition of glycogen synthase kinase 3 enzymes and thus the transcriptional activation functions of the T-cell factor/lymphoid enhancement factor (TCF/LEF) transcription factors ${ }^{2}$. In contrast to CBCs, quiescent ISCs divide infrequently, are located above the Paneth cell and $\mathrm{CBC}$ niche, and are Wnt independent ${ }^{3-6}$. Both ISC types can generate all intestinal epithelial cell types, a function apparently provided primarily by CBCs, with quiescent ISCs dividing to generate new CBCs on the occasion of their loss ${ }^{5,6}$. Frequent cell divisions by CBCs put them at risk for replication-dependent telomere shortening. To guard against such shortening, CBCs express high levels of telomerase, which at its core comprises a polymerase (TERT) and associated RNA template (TR) ${ }^{2,7}$.

People with genetic mutations causing partial deficiencies in telomerase activity typically display a spectrum of pathologies collectively termed dyskeratosis congenita (DC), which includes defects in highly proliferative tissues, including bone marrow and the epithelia of the skin and gastrointestinal tract, and in tissues that proliferate in response to injury, including liver and lung ${ }^{8}$. The premature loss of telomeres in DC can be modelled in mice by genetic inactivation of telomerase, for example, $m$ Tert $^{-/-}$or $m T R^{-/-}$mutants. Lab strains of mice have long telomeres, and thus initial generations lacking telomerase (for example, G1 or G2 $m T R^{-}{ }^{-}$mutants) are relatively unaffected by telomere shortening 9,10 . However, critical telomere shortening in lategeneration mice yields pathology primarily in proliferative tissues such as intestine, characterized by elevated levels of apoptosis in the crypt epithelium ${ }^{10-12}$. Recent reports indicate reduced expression of Wnt pathway genes in the intestinal epithelium of such mice ${ }^{13,14}$, but the full extent and functional importance of these Wnt pathway deficits have not been explored.

Here we demonstrate broad downregulation of canonical Wnt pathway gene expression in the intestinal crypt epithelium and underlying stroma of late-generation $m T R^{-/-}$mice. These changes appear to reflect a regulatory response to critical telomere shortening, and involve upregulation of the miR34a microRNA (miRNA). We further show that Wnt pathway agonists rescue intestinal pathology, gene expression, and most remarkably, telomere capping itself, in a fashion that does not involve telomere lengthening. These findings reveal a positive feedback loop between telomere capping and Wnt pathway activity, and are distinct from previous suggestions of connections between Wnt and the catalytic subunit of telomerase, TERT (refs 15-17). They also raise the possibility of novel therapeutic approaches to diseases with underlying telomere dysfunction.

\section{Results}

Wnt gene expression declines in late-generation $m T^{-/-}$mice. We examined CBC marker gene expression ${ }^{18}$ in successive generations of $m T R$-deficient mice, (rather than mTert mutants, to avoid perturbing potential non-telomeric roles of mTert (ref. 19)). Expression of the Wnt pathway target genes Ascl2 and Sox9 were decreased in late-generation (generation 4, or G4) $m T R^{-1-}$ mice (Fig. 1a,b). Minimal changes in G2 mice indicate that the decreased expression is caused by dysfunctional telomeres rather than telomerase deficiency per se. Wnt ligands produced by stromal and Paneth cells provide support essential to CBC function $^{20,21}$. Because Ascl2 and Sox9 are direct targets of the
Wnt signalling pathway, we hypothesized that the diminished expression in G4 $\mathrm{mTR}^{-/-}$crypts reflects a defect in the Wnt pathway caused by telomere dysfunction. Crypt epithelium and underlying stroma were each isolated from wild type (WT) and G4 mice (Supplementary Fig. 1) and used for messenger RNA expression profiling. Indeed, among the sets most significantly downregulated in mutant crypts are those with genes similarly downregulated on deletion of the Wnt signalling mediator $\beta$-catenin, or altered with perturbation of other Wnt pathway components (Fig. 1c,d; Supplementary Figs 2 and 3b,d; Supplementary Table 1). Altered expression was confirmed by quantitative PCR for a number of the Wnt pathway and target genes, including Wnt3, Lrp6 and Lgr5, the last of which is not only a Wnt target and $\mathrm{CBC}$ marker, but also a key Wnt receptor component (Fig. 1d).

Some degree of Wnt pathway gene downregulation may stem from CBC losses, but these cannot explain most of our observations. In particular, staining for lysozyme-positive Paneth cells revealed neither loss of these cells nor of interposed cells (Fig. 1e-g), where CBCs normally reside. Thus, diminished expression of $\mathrm{CBC}$ marker genes apparently reflects a change in state, rather than presence, of CBCs. Furthermore, the decline in Wnt3 transcripts (Fig. 1d) cannot be explained by cell loses because Paneth cells are the only intestinal source of Wnt3 (ref. 21). Additionally, apoptosis is not significantly elevated in the stroma but changes consistent with decreased Wnt pathway activity were also found, including upregulation of SFRP Wnt antagonists (Fig. 2a; Supplementary Fig. 3a,c). There were also no significant changes in the expression of genes associated with quiescent ' +4 ' ISCs, including Bmil, mTert, Hopx and Lrig1 (Supplementary Fig. 2c), consistent with maintenance of Wntindependent gene expression. Collectively, the data from epithelium and stroma indicate broad Wnt pathway suppression in G4 $m T R^{-/-}$intestinal tissues.

Based on defects in G1 TTert $^{-/}$- but not G1 mTR ${ }^{-/-}$mice, it was suggested ${ }^{15}$ that Tert but not TR regulates Wnt signalling, independent of its telomere-lengthening function. This idea has been questioned ${ }^{22,23}$, and moreover is quite different from our findings. First, our mice are genetically deficient for $m T R$ and not $m$ Tert, and indeed mTert transcripts were not decreased in G4 $m T R^{-1}-$ crypts (Supplementary Fig. 2c). Second, suppression of Wnt pathway gene expression progressed with successive generations (Fig. 1 and Supplementary Fig. 4b,c), confirming that telomere dysfunction, rather than telomerase deficiency per se causes the suppression.

miR34a contributes to diminished Wnt pathway gene expression. We considered how Wnt pathway components are broadly downregulated in $m T R^{-/-}$mutants. Dysfunctional telomeres elicit p53-dependent DNA damage responses, and p53-activated upregulation of miR34a has recently been implicated in the suppression of multiple Wnt pathway genes ${ }^{24}$. Indeed, we detected p53 activation (as measured by p21 expression) and a significant increase in miR34a expression in $m T R^{-/}$crypts (Fig. 2b; Supplementary Fig. 4a). Further, miRNA profiling indicated miR34a was the most upregulated canonical miRNA in mutant epithelium (Supplementary Table 2). We therefore tested interbred mice with genetic deficiencies in miR34a and mTR (Supplementary Fig. 5a) to test the hypothesis that miR34a loss would rescue defects associated with telomere dysfunction. Consistent with this idea, significantly more G4 mice survived to weaning at 3 weeks of age if they lacked miR34a (Supplementary Fig. 5b). Moreover, such G4 $m T R^{-/-} m i R 34 a^{-1-}$ mice exhibited significantly decreased crypt apoptosis, improved telomere capping as demonstrated fewer telomere-dysfunction-induced foci (TIFs; measured by 
a

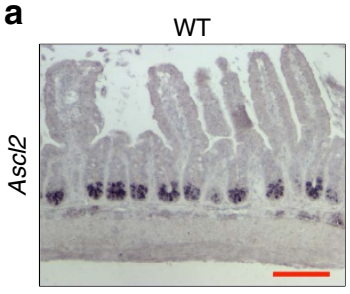

b

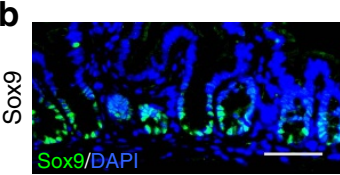

C

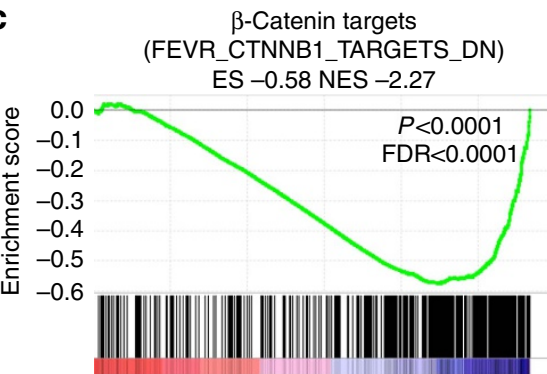

Rank in ordered data set

( $G 4$ versus WT)

e
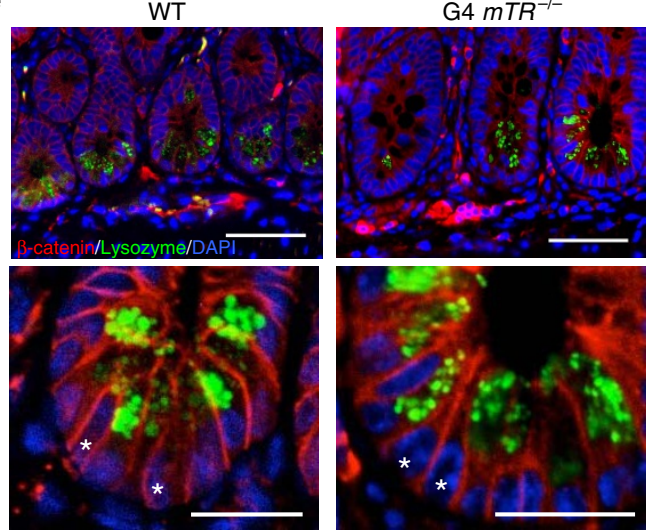

$\mathrm{G} 2 m T R^{-1-}$
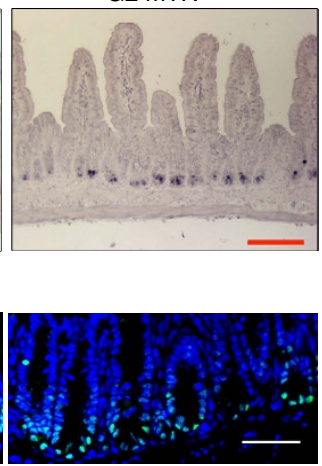

d
G4 $m T R^{-1}$
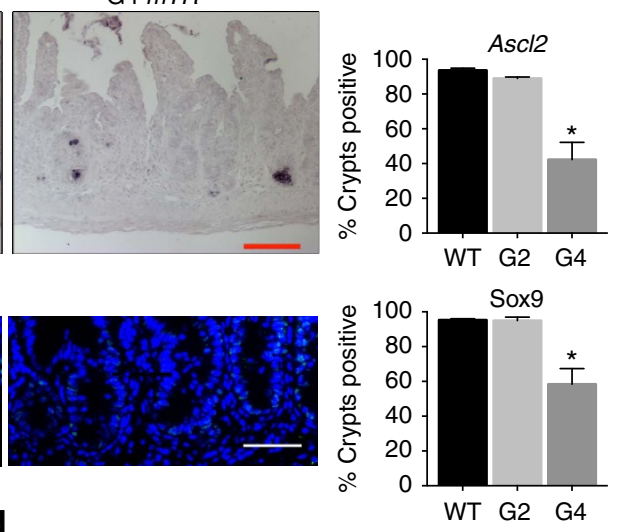

\section{d}

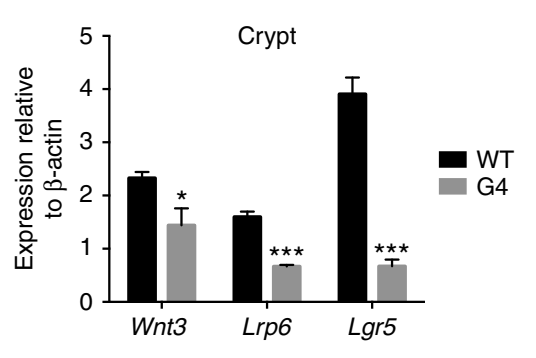

f
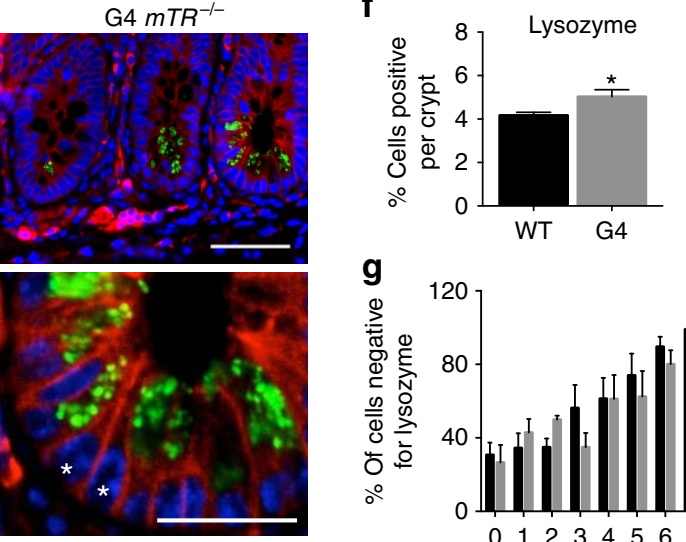

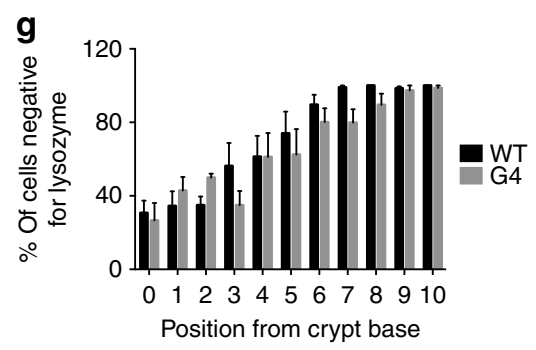

Figure 1 | Defects in small intestinal CBC stem cell and Paneth cell gene expression in late-generation $\boldsymbol{m T R}^{-/-}$mice. (a) Representative images of in situ hybridization for Ascl2 transcripts in ileum from WT, G2 and G4 $m T R^{-/-}$mice and quantitation of crypts positive for staining (right; $n=3$ ); ${ }^{*} P<0.05$. Scale bars, $100 \mu \mathrm{m}$. (b) Representative images of Sox9 immunostaining in ileum from WT $(n=5), 2$ nd generation $(G 2, n=3)$ and 4 th generation $(\mathrm{G} 4, n=4) \mathrm{mTR}^{-/}-$mice and quantitation of crypts positive for staining (right); ${ }^{\star} P<0.05$. Scale bars, $50 \mu \mathrm{m}$. (c) Gene Set Enrichment Analysis (GSEA) revealed G4 $m T R^{-/-}$ileal crypts to have significantly reduced expression of genes downregulated in $\beta$-catenin knockout mouse crypts (FEVR_CTNNB1_TARGETS_DN) $(n=3)$. Enrichment score $(E S)$, normalized enrichment score $(N E S), P$ value and false discovery rate (FDR) are as described ${ }^{50}$ (see 'Methods' section). (d) Quantitative reverse transcription PCR (qRT-PCR) analyses of gene expression for the Wnt ligand (Wnt3), Wnt co-receptor (Lrp6) and Wnt target gene and co-receptor (Lgr5) in WT and G4 mTR ${ }^{-/-}$crypts $(n=5)$. Also, note that Msi1 protein expression is unchanged (Supplementary Fig. 2d). ${ }^{\star} P<0.05$ and ${ }^{\star \star \star} P<0.005$. (e) Immunostaining for Paneth cell lysozyme in WT and G4 $m T R^{-/}-$ileum. Enlarged insets show examples of cells at the normal CBC location between lysozyme-positive Paneth cells (asterisks). $\beta$-catenin staining marks cell peripheries. Scale bars, $50 \mu \mathrm{m}$ (top), $25 \mu \mathrm{m}$ (bottom). (f) Quantitation of Paneth cells as measured by lysozyme staining and ( $\mathbf{g}$ ) of cells intercalated between Paneth cells from WT and G4 $\mathrm{mTR}^{-/-}$crypts $(n=6) ;{ }^{\star} P<0.05$. All error bars reflect s.e.m., and $P$ values reflect unpaired two-tailed Student's $t$-tests.

colocalization of telomeres with 53BP1 (ref 25)), and a trend towards fewer anaphase bridges, indicative of fewer telomeretelomere fusions (Fig. 2c-e; Supplementary Fig. 5c). In addition, miR34a inhibition in cultured intestinal organoids derived from $m T R^{-/-}$mice restored crypt budding and expression of the Wnt pathway target genes Lgr5 and Trf2 (Supplementary Fig. 6, and see below). To extend published evidence for targeting of Wnt pathway genes by miR34a (Supplementary Table 3), we examined regulation of Lgr4, which cooperates with Lgr5 to support CBCs (ref. 26). miR34a deletion increased expression in G4 fibroblasts of a luciferase reporter fused to the Lgr4 $3^{\prime} \mathrm{UTR}$, containing four miR34a target sequences, illustrating suppression of the Wnt pathway target by miR34a in G4 $\mathrm{mTR}^{-/}$cells (Supplementary Fig. 7). 
a

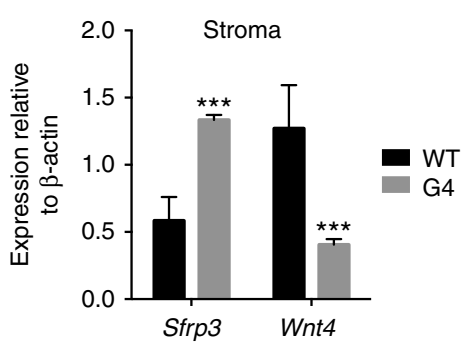

b

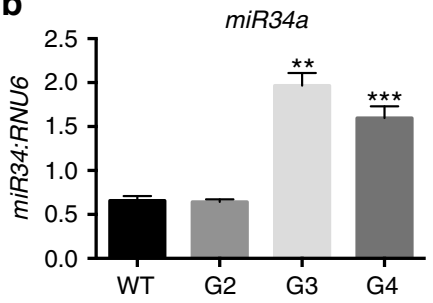

C
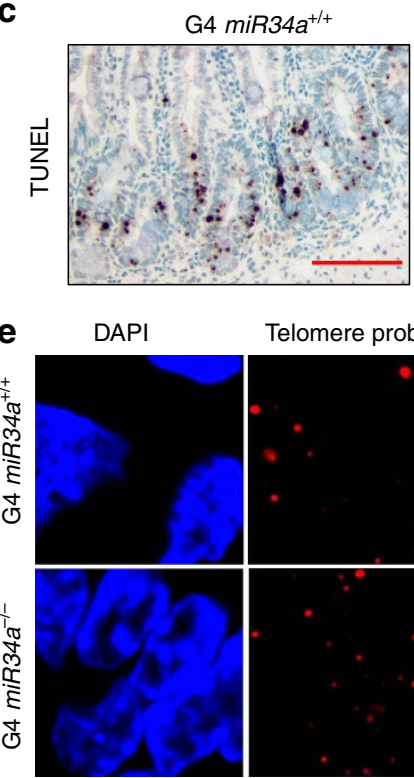
Telomere probe

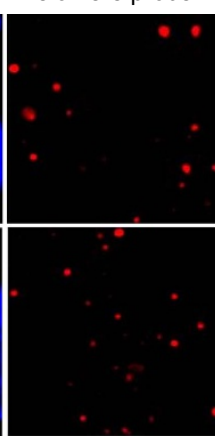

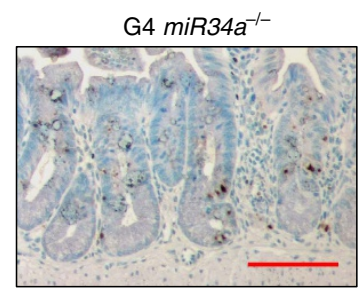
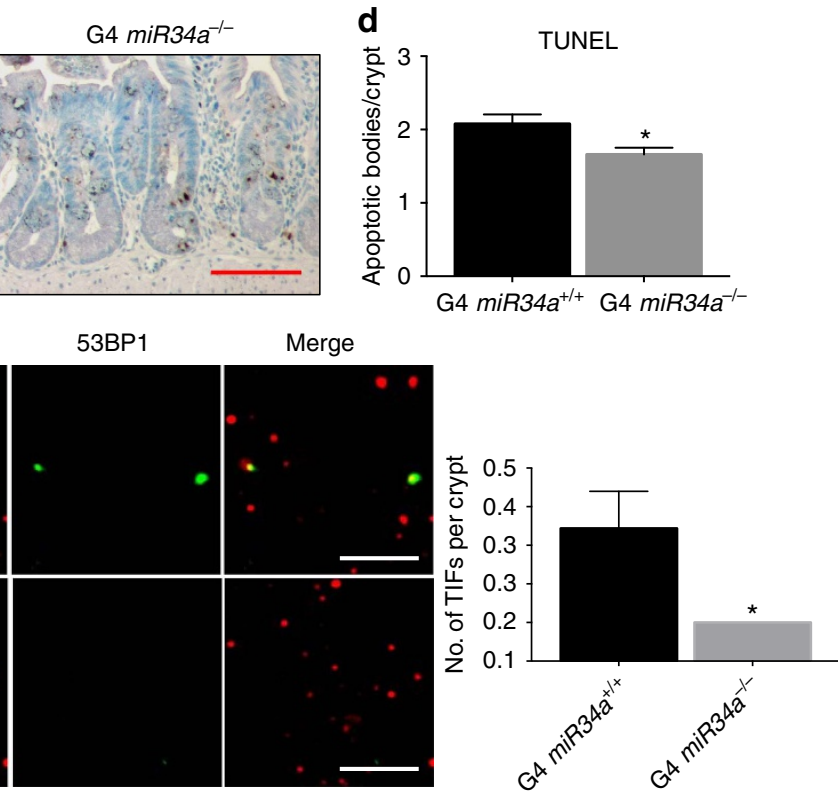

Figure 2 | G4 $\mathbf{m T R} \mathbf{R}^{-/-}$mice have reduced expression of pro-Wnt pathway genes in ileal crypts and stroma. (a) Quantitative reverse transcription PCR (qRT-PCR) analyses of gene expression for the Wnt ligand (Wnt4) and Wnt receptor inhibitor (Sfrp3) in WT and G4 mTR ${ }^{-/-}$stroma ( $\left.n=4\right)$. (b) qRT-PCR of miR34a in WT, G2, G3 and G4 mTR ${ }^{-/-}$crypts ( $n=4$ for WT, G2, G3; $n=7$ for G4). (c) Representative images showing apoptotic changes by TUNEL staining of ileum of G4 mTR ${ }^{-/-}$miR34a $a^{+/+}$mice and G4 mTR ${ }^{-/-}$miR34a- $a^{-}$mice. Scale bar, $50 \mu \mathrm{m}$. (d) Quantitation of TUNEL-positive apoptotic bodies per crypt $(n=6)$. (e) Representative images showing reduced telomere dysfunction as measured by TIFs in G4 mTR ${ }^{-/-}$miR34a $a^{-/-}$mice compared to littermate $\mathrm{G} 4 \mathrm{mTR}{ }^{-/-}$miR34a $a^{+/+}$mice $(n=4)$. Quantitation of TIFs (right). Scale bar, $5 \mu \mathrm{m}$. All error bars reflect s.e.m., and $P$ values reflect unpaired two-tailed Student's $t$-tests, with ${ }^{\star} P<0.05,{ }^{\star \star} P<0.005$ and ${ }^{\star \star \star} P<0.0005$.

Rescue telomere defects by Wnt pathway agonists. We next asked whether Wnt pathway agonists could ameliorate pathology by restoring the niche environment. Indeed, subcutaneous injection of Rspo1, which selectively potentiates intestinal Wnt signalling via the Lgr5 co-receptor ${ }^{27}$, suppressed apoptosis and led to elevated Ascl 2 transcript and Sox9 protein levels (Fig. 3a-c). Furthermore, crypt apoptosis and Ascl2 expression were rescued in G3 $m T R^{-1-}$ mice fed chow containing lithium, which is a glycogen synthase kinase 3 inhibitor ${ }^{28}$, and this rescue persisted for at least 44 days (Supplementary Figs 8 and 9). As a complementary approach, we tested effects of Rspol and the selective glycogen synthase kinase 3 inhibitor CHIR99021 (ref. 29) on G4 $\mathrm{mTR}^{-/-}$intestinal organoid cultures. Both Rspo1 and CHIR99021 yielded dose-dependent improved mutant organoid crypt morphology, and CHIR99021 suppressed transcripts of Noxa (a mediator of p53-induced apoptosis) and rescued Lgr5 transcript levels (Fig. $4 \mathrm{a}-\mathrm{e}$ ). In sum, these in vivo and in vitro data establish roles for Wnt pathway agonists in rescuing abnormalities in late-generation $m T R^{-/-}$crypts related to morphology, apoptosis and $\mathrm{CBC}$-related gene expression.

Wnt pathway activity is known to enhance intestinal epithelial cell proliferation, and we observed increased Ki67-positive cells in the transit amplifying, but not crypt bases, of Rspol and lithiumtreated G4 $m T R^{-1}-$ mutants (Supplementary Fig. 10). Without telomerase activity, enhanced proliferation should only exacerbate telomere shortening and dysfunction, making rescue seemingly paradoxical. We therefore investigated whether rescue might be associated with improved telomere capping. We scored TIFs and found a significant decrease in the number in crypts of G4 $\mathrm{mTR}^{-1-}$ mice treated with Rspol, as well as decreased anaphase bridges, indicating fewer telomere fusions (Fig. 3d,e). Telomere lengths in crypt epithelial cells of lithium or Rspo1treated G4 $m T R^{-/-}$mice were not longer than those of untreated G4 $m T R^{-/}$mice (Supplementary Fig. 11), ruling-out telomerase-independent lengthening. In fact, telomeres appeared to shorten in lithium-treated mice, consistent with enhanced proliferation due to improved telomere capping. In the complete absence of telomerase, additional telomere shortening would presumably lead eventually to stem cell exhaustion. Nonetheless, long-term (44 day) treatment with lithium maintained telomere capping, suppressed apoptosis and anaphase bridges, and enhanced Ascl2 expression (Supplementary Fig. 9). Therefore, Wnt pathway agonists can restore capping of short telomeres without net lengthening in the absence of telomerase.

Telomere capping depends not only on telomere length but also on several key shelterin proteins, including Trf2 and Potla (ref. 30). Trf2 was shown recently to be upregulated by the Wnt pathway in human cells and murine intestinal tissue, and elevated Trf2 can rescue uncapped telomeres in senescing human fibroblasts and following $\beta$-catenin inhibition in cancer 
a

$\mathrm{G} 4 m T R^{-/-}$
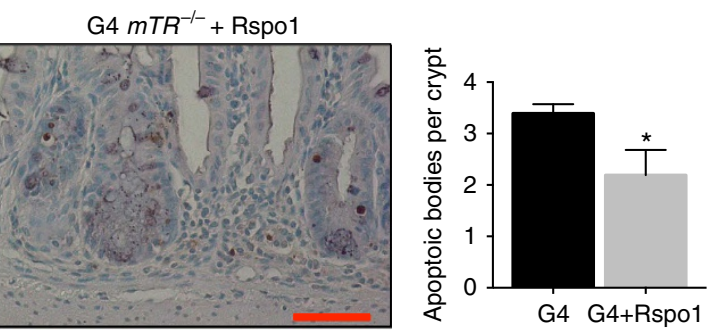

b
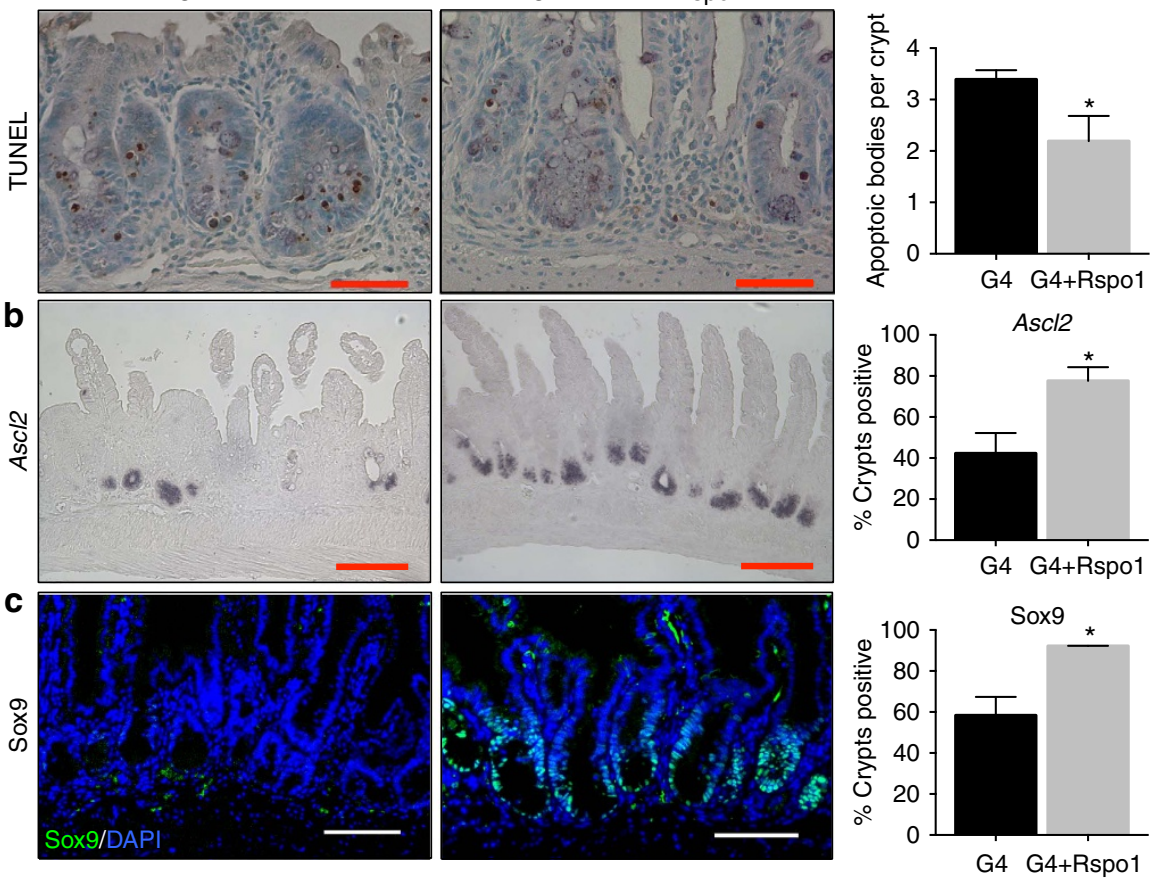

d
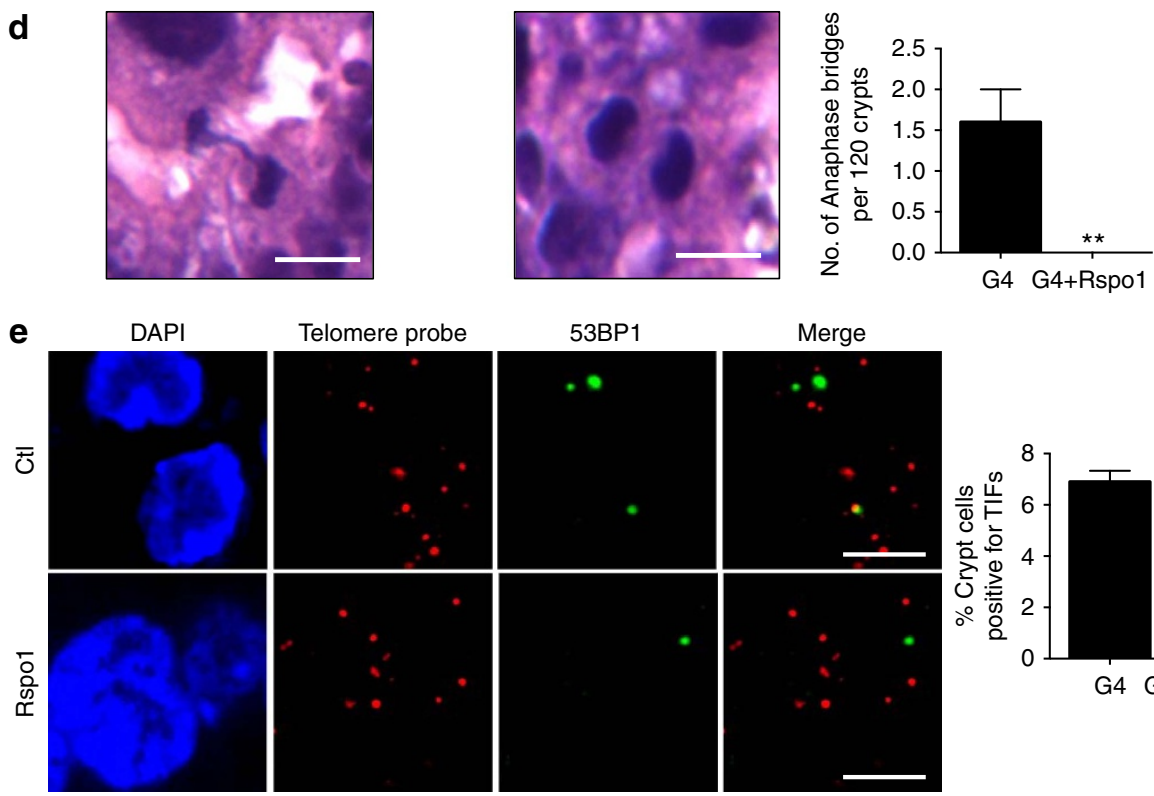

Telomere probe
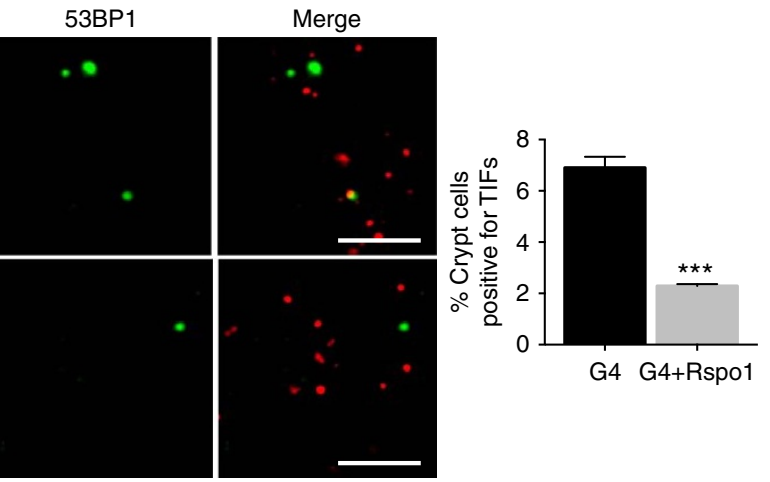

Figure 3 | Enhanced Wnt signalling rescues G4 $\mathbf{m T R ^ { - / }}$ - crypt dysfunction and telomere uncapping in vivo. (a) Representative images showing apoptotic changes by TUNEL staining in intestinal crypts of G4 mTR I $^{-}$- mice treated for 8 days with subcutaneous injections of R-spondin1 (Rspo1) ( $n=4$ Rspo1-treated and 5 littermate controls). Scale bars, $50 \mu \mathrm{m}$. (b) Representative image showing Ascl2 transcripts differences in Rspo1-treated and control G4 mTR ${ }^{-/-}$mice $(n=3)$. Scale bars, $100 \mu \mathrm{m}$. (c) Representative images showing Sox9 protein differences in Rspo1-treated and control G4 $m T R^{-/-}$mice ( $n=4$ control and 3 Rspo1-treated). Scale bars, $50 \mu \mathrm{m}$. (d) Representative images showing reduced telomere dysfunction as measured by anaphase bridges in Rspo1-treated G4 mTR ${ }^{-/-}$mice (left, untreated; middle, Rspo1-treated). Quantitation of anaphase bridges (right). 120 crypts per mouse from 5 control G4 mice and 4 Rspo1-treated G4 mice were surveyed for anaphase bridges. Scale bars, $10 \mu \mathrm{m}$. (e) Representative images and quantitation (right) showing reduced telomere dysfunction as measured by TIFs in Rspo1-treated G4 mTR ${ }^{-/-}$mice $(n=3)$. Scale bars, $5 \mu$ m. All error bars reflect s.e.m., and $P$ values reflect unpaired two-tailed Student's $t$-tests, with ${ }^{\star} P<0.05$, ${ }^{\star \star} P<0.005$ and ${ }^{\star \star \star} P<0.0005$.

cells $^{31,32}$. We analysed published data sets and found additional evidence that shelterin genes are regulated by Wnt, including APC- and $\beta$-catenin-dependent regulation of $\operatorname{Tr} 2$, and TCF4 binding to TRF1, TRF2 and POT1 (but not TPP1 and RAP1) in humans (Supplementary Fig. 12) ${ }^{33,34}$. Consistent with these observations, levels of $\operatorname{Trf1}, \operatorname{Trf} 2, \operatorname{Pot} 1 a / b$ (and not Tpp1 and Rap1) transcripts were reduced significantly in late-generation $m T R^{-1-}$ crypts (Supplementary Figs $4 \mathrm{c}$ and 12a). Moreover, CHIR99021 provided dose-dependent upregulation of $\operatorname{Trf2}$, Pot1a, Tin2 and Trf1 transcripts in G4 crypt cultures (Fig. $4 \mathrm{e}$ and Supplementary Fig. 13). Similar results were obtained in human patient-derived fibroblasts with premature telomere uncapping caused by telomerase deficiency (dykeratosis congenita, DC) or telomere replication defects (Werner 
a

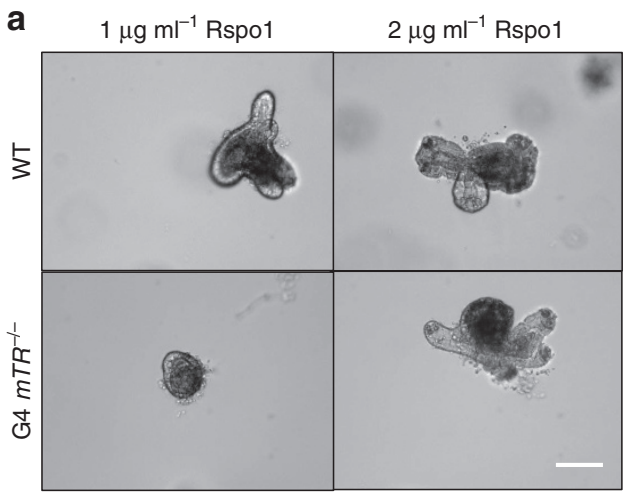

C

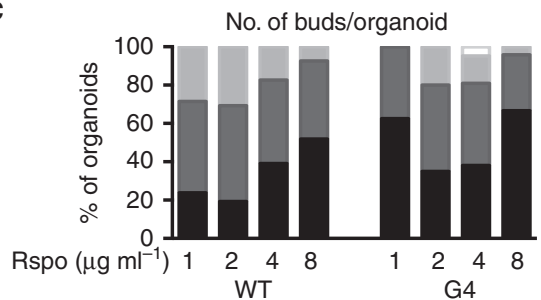

b
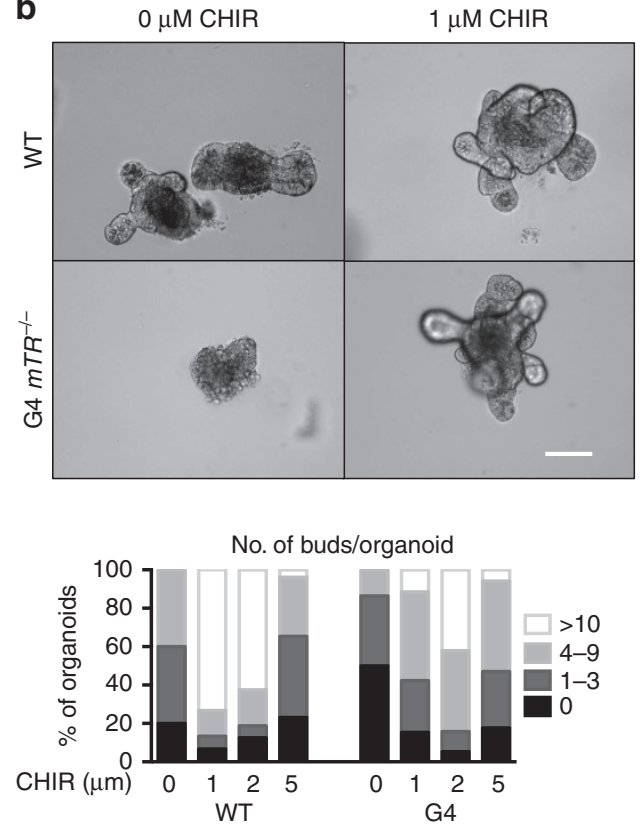

d

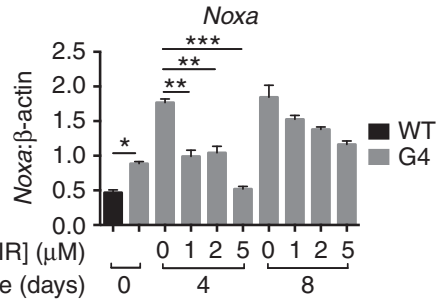

e
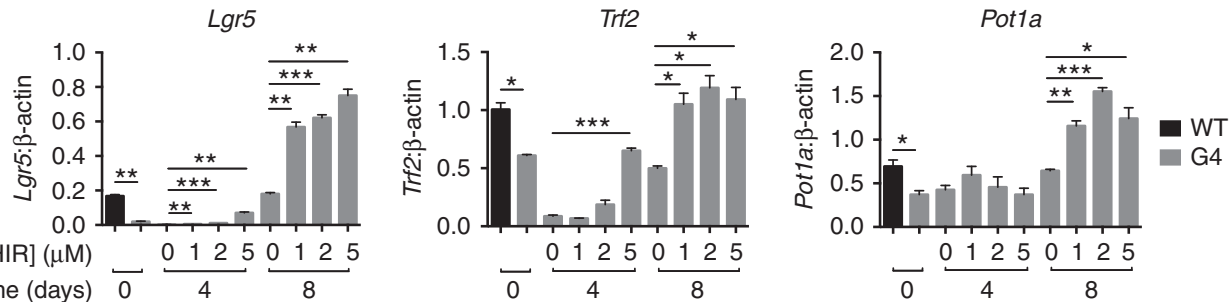

Figure 4 | Wnt pathway agonists rescue survival and morphology of cultured $\mathbf{G 4} \mathbf{m T R ^ { - / }}$ - intestinal organoids. (a,b) Representative micrographs showing intestinal organoids from WT and G4 $\mathrm{mTR}^{-/-}$mice cultured under standard conditions ( $1 \mu \mathrm{g} \mathrm{ml}^{-1}$ Rspo1) or augmented with elevated levels of (a) Rspo1 or (b) the glycogen synthase kinase 3 inhibitor CHIR99021. Under standard culture conditions, WT organoids are long-lived and appear as roughly spherical epithelium surrounding a central cavity representing the lumen, and have crypt buds radiating outward from their peripheries. G4 $m T R-/$

- organoids were prone to degeneration and had fewer crypts under basal conditions, but could be rescued with slightly elevated Rspo1 or CHIR99021. Scale bars, $100 \mu \mathrm{m}$. (c) The number of buds per organoid was quantified and grouped accordingly $(0,1-3,4-9,>10$ buds per organoid), and expressed as a percentage of all organoids. Note that the well-established inhibition of crypt budding by supraphysiologic levels of Wnt pathway activity is shifted to higher doses of Rspo and CHIR in the G4 organoids, consistent with a basal Wnt pathway defect. (d) Quantitative reverse transcription PCR (qRT-PCR)

measurement of Noxa transcripts, a mediator of p53-activated apoptosis, in WT and G4 $\mathrm{mTR}^{-/-}$crypts at $t=0$, and treated with increasing doses of CHIR99021 at days 4 or $8(n=3) ;{ }^{*} P<0.05,{ }^{\star \star} P<0.01$ and ${ }^{\star \star \star} P<0.0001$. (e) Dose- and time-dependent increase of Lgr5, Tfr2 and Pot7a transcript expression as measured by qRT-PCR in WT and G4 mTR ${ }^{-/-}$crypts cultured with CHIR99021 for 4 or 8 consecutive days $(n=3)$. For all panels, ${ }^{\star} P<0.05$, ${ }^{\star \star} P<0.005$ and ${ }^{\star \star \star} P<0.0005$. Also, note that miR34a inhibition similarly rescued organoid budding, Lgr5 and Trf2 expression (Supplementary Fig. 6). All error bars reflect s.e.m., and $P$ values reflect unpaired two-tailed Student's $t$-tests.

syndrome; Supplementary Fig. 14). Thus telomere capping via Wnt pathway activation may be explained by upregulation of shelterin components.

Our study reveals that loss of telomere capping can lead to broad suppression of Wnt pathway activities in both intestinal epithelium and stroma, and moreover, that enhancement of Wnt pathway activity can rescue telomere capping, apoptosis and the ISC gene expression program. Importantly, components of the Wnt pathway are themselves pathway targets (for example, Lgr5, encoding a Rspo1-responsive component of the Wnt receptor complex $)^{18}$, providing a mechanism by which the effects of Wnt pathway agonists can be amplified to restore the pathway. Improved telomere capping is not explained by telomere lengthening, but rather is associated with restoration of expression of key shelterin genes, including $\operatorname{Trf} 2$ and Pot1a. Whether Wnt pathway agonists sensitize or protect intestine from ionizing radiation is debated ${ }^{14,35,36}$. Regardless, upregulation of shelterin proteins would be expected to have a salutary effect for 
shortened telomeres but not radiation-induced genome-wide breaks, consistent with the clear benefits of enhanced Wnt pathway activity in $m T R^{-/-}$mutants. Our findings raise the possibility that disorders in which telomere dysfunction plays a pathogenic role (for example, DC or ulcerative colitis) ${ }^{37-39}$ might be treated by Wnt pathway agonists. The haematopoietic system is often severely affected in patients with telomere diseases, and since canonical and noncanonical Wnt signalling play complex roles in haematopoietic homeostasis ${ }^{40-42}$, further studies are warranted to establish effects Wnt agonists have on the haematopoietic system in the setting of telomere dysfunction. However, lithium has been used for many decades to treat bipolar disorder, and leucocyte telomere lengths and TERT expression in treated patients positively correlate with duration of therapy ${ }^{43,44}$. This therapeutic approach might raise concern given that hyperactivation of Wnt signalling can drive cancer, but a recent large-scale case-control study found no association between longterm lithium use and risk of colorectal adenocarcinoma ${ }^{45}$. Furthermore, the elevated cancer rates of DC patients indicate that excessive telomere shortening due to telomerase deficiency is itself carcinogenic, and therefore improved telomere capping may actually suppress cancer in such individuals. Wnt agonists may be of particular benefit in patients with such telomere diseases because they may not only upregulate key shelterins and improve telomere capping at any given telomere length (as our current study demonstrates), but due to their ability to effect TERT upregulation $^{16,17}$ they may also improve telomere capping via lengthening.

\footnotetext{
Methods

Study design. Histologic comparisons of WT, G2, G4 $m T R^{-/-}$and G4 $m T R^{-/-} m i R 34 a^{-/-}$mice were made between groups each having similar average age, and all mice were younger than 12 months. RNA samples for microarray analysis and quantitative reverse transcription PCR validation were obtained from WT and G4 $m T R^{-1-}$ mice aged 7-8 months. For each of the lithium and Rspol treatment experiments, littermates were divided equally into the control and treatment groups. Comparisons were made between littermates to minimize differences in inherited telomere lengths. For cultured crypts experiments, G4 $\mathrm{mTR}^{-/-}$mice aged 3 months or younger were used because survival of crypts dropped precipitously beyond this age.
}

Mice. All mice were on the C57BL/6 J background, and $m T R^{+/-}$mice were crossed to generate G1 $\mathrm{mTR}^{-1-}$ mice, which were crossed to generate G2 mice, and so forth ${ }^{9} . m T R$ mutant mice were obtained from the laboratory of R. DePinho and backcrossed 12 times onto the C57Bl/6 J background, and miR34a ${ }^{-/-}$

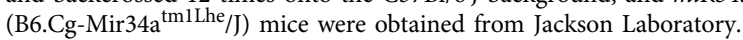
Comparisons were made between age- and sex-matched mice except in the longterm lithium treatment experiment where sex-matching was not possible (Supplementary Fig. 9). In addition, sex-matching was not always possible for comparisons between G4 $m T R^{-1-} m i R 34 a^{-1-}$ and G4 $m T R^{-1-} m i R 34 a^{+1+}$ mice. Mice for the microarray experiment were females and ranged from 6 to 8 months old. Mice for the Rspol and lithium treatments were males and ranged from 6 to 8 months old. Crypts were isolated from mice 1 to 3 months of age for organoid culture experiments. The breeding scheme for generating miR34a ${ }^{-/-}$ onto the different generations of $m \mathrm{TR}^{-/-}$mice is outlined in Supplementary Fig. 5a. All studies are approved by the University of Pennsylvania Institutional Animal Care and Use Committee. The mice were housed in a standard animal care room with 12:12-h light-dark cycle with free access to food and water. For the short-term 10-day lithium treatment, mice were given an ad libitum lithium-chow diet of $0.212 \%$ lithium chloride-supplemented chow (Harlan Teklad, Madison, WI, USA) for 3 days, followed by $0.4 \%$ lithium chloride-supplemented chow for 7 days. For the long-term 44-day lithium treatment, mice were given 4 consecutive cycles of $0.212 \%$ lithium-chow for 4 days, followed by $0.4 \%$ lithium-chow for 7 days. Lithium-chow-fed mice were given a supplemental source of drinking water containing $1.5 \%(\mathrm{w} / \mathrm{v})$ sodium chloride to counteract potential toxicities of lithium. For Rspol experiments, mice are injected at $4 \mu \mathrm{g}$ Rspol (in PBS, or PBS alone for controls) per gram body weight subcutaneously daily for 8 days. Rspo1 was expressed and purified as described ${ }^{46}$. Briefly, HEK293T cells stably transfected with an hRspol expression vector were cultured in suspension, protein was precipitated from the culture medium with ammonium sulfate, purified by column chromatography, dialyzed into PBS, $0.22 \mu \mathrm{M}$ filtered, and stored at $-80^{\circ} \mathrm{C}$.
Tissue histology. Standard $5-\mu$ sections were cut from formalin-fixed and paraffinembedded samples. In situ hybridization for Ascl2 was as described ${ }^{18}$. Briefly, for in situ hybridization, formalin-fixed and paraffin-embedded samples were deparaffinized, rehydrated, treated with $\mathrm{HCl}$, digested in proteinase $\mathrm{K}$ solution, refixed with formaldehyde, treated in acetic anhydride solution, and hybridized with a digoxigenin labelled RNA probe specific for Ascl2. After washes with $2 \times$ salinesodium citrate (SSC) and $50 \%$ formamide $/ 2 \times$ SSC, sections were incubated overnight at $4{ }^{\circ} \mathrm{C}$ with alkaline phosphatase-conjugated anti-digoxigenin antibodies (Roche \#11093274910 at a 1:2,000 dilution, that is, 0.375 units per ml), and developed using nitro blue tetrazolium/5-bromo-4-chloro-3-indolyl-phosphate (NBT/BCIP) solution. For immunofluorescent detection of Sox9, sections were treated as follows, at room temperature unless stated otherwise. Sections were deparaffinized by heating to $60^{\circ} \mathrm{C}$ for $15 \mathrm{~min}$, washed $3 \times 5 \mathrm{~min}$ in xylene, rehydrated in a series of ethanol solutions $(100 \%, 95 \%, 90 \%$ and $70 \%$ ethanol), washed in water and in $0.1 \%$ Tween-20 for 1 min each, steamed in antigen unmasking solution (Vector Laboratories H-3300) for $15 \mathrm{~min}$, washed in phosphatebuffered saline containing $0.2 \%$ Tween-20 (PBST) for 5 min, blocked in $4 \%$ BSA in PBST for $30 \mathrm{~min}$, incubated with rabbit anti-Sox9 antibodies (Millipore AB5535 at $1.7 \mu \mathrm{g} \mathrm{ml}^{-1}$ ) overnight at $4^{\circ}$ in $1 \%$ BSA in PBST, washed three times for $5 \mathrm{~min}$ in PBST, incubated with Alexa 488-conjugated goat anti-rabbit antibody (Invitrogen A-11034 at $2.5 \mu \mathrm{g} \mathrm{ml}^{-1}$ ) for 2 hours, washed three times for five minutes in PBST including $0.2 \mu \mathrm{g} \mathrm{ml}^{-1} 4,6$-diamidino-2-phenylindole (DAPI) in the penultimate wash, and mounted in ProLong Gold antifade reagent (Thermo Fisher Scientific). For immunohistochemical detection of Ki67, sections were deparaffinized, rehydrated and subjected to antigen unmasking in the same fashion as for Sox 9 staining, and were then treated with $2.25 \%$ hydrogen peroxide for $15 \mathrm{~min}$, washed in water and PBST for 5 min each, blocked with Avidin D reagent (Vector Laboratories SP-2001), washed in PBST, blocked with 4\% BSA in PBST for 30 min, incubated with rabbit anti-Ki67 antibody (Abcam Ab16667 at 1:100), washed three times for $5 \mathrm{~min}$ in PBST, incubated with biotinylated anti-rabbit antibody (Vector Laboratories BA-1000 at $7.5 \mu \mathrm{g} \mathrm{ml}^{-1}$ ) for $30 \mathrm{~min}$, washed in PBST 3-5 min, stained with the Vector Laboratories ABC and DAB reagents (PK-6200 and SK-4100), counterstained with Gill \#2 haematoxylin (Thermo Fisher Scientific), dehydrated in an ethanol series (70-100\%), cleared in xylene, and mounted in Cytoseal XYL (Thermo Fisher Scientific 8312-4). Terminal deoxynucleotidyl transferase dUTP nick-end labeling (TUNEL) assays were performed using the ApopTag Peroxidase In Situ apoptosis Detection Kit (Chemicon). Images within each set of staining were taken with identical settings on a Nikon Eclipse E600 microscope. Images were taken with identical settings, and brightness/contrast was adjusted post-capture in a linear fashion and equally for all samples. 120 crypts per mouse from haematoxylin and eosin stained sections were surveyed for the presence of anaphase bridges.

TIF and telomere quantitative fluorescence in situ hybridization analyses. Tissue sections were hybridized with a Cy3-labelled PNA telomere repeat probe (Panagene, $\left(5^{\prime} \text {-CCCTAA- } 3^{\prime}\right)_{3}$ ) and anti-53BP1 antibodies (Novus, NB100-304) as described with slight modifications ${ }^{47}$. Briefly, formalin-fixed and paraffin-embedded tissue sections were deparaffinized with xylene, rehydrated with 95,85 and $70 \%$ ethanol series, rinsed with $\mathrm{H}_{2} \mathrm{O}$ and $0.1 \%$ Tween-20, and subjected to antigen retrieval by covering with sodium citrate buffer (Vector labs, H-2200) and a cover slide and then steaming for $15 \mathrm{~min}$. Sections were then washed with PBST $(0.2 \%$ Tween-20 in PBS), and then blocked in $4 \% \mathrm{BSA} / \mathrm{PBST}$ for $30 \mathrm{~min}$ at $37^{\circ} \mathrm{C}$, incubated with rabbit anti-53BP1 antibodies $\left(10 \mu \mathrm{g} \mathrm{ml}^{-1}\right)$ for $2 \mathrm{~h}$ at $37^{\circ} \mathrm{C}$, washed with PBST, incubated and Alexa Fluor 488-conjugated goat anti-rabbit antibodies (Invitrogen, $\mathrm{A}-11034,4 \mu \mathrm{g} \mathrm{ml}^{-1}$ ) for $1 \mathrm{~h}$ at $37^{\circ} \mathrm{C}$. After washing with PBST, slides were fixed with $4 \%$ paraformaldehyde in PBS at room temperature for $20 \mathrm{~min}$, followed by quenching of the formaldehyde with $0.25 \mathrm{mM}$ glycine. The sections were subsequently dehydrated with ethanol and air dried. Cy3-conjugated telomerespecific PNA probe was applied in hybridization mix (per $100 \mu \mathrm{l}$, mix $70 \mu \mathrm{l}$ of freshly deionized formamide, $15 \mu$ PNA buffer $(80 \mathrm{mM}$ Tris-Cl pH 8, $33 \mathrm{mM} \mathrm{KCl}, 6.7 \mathrm{mM}$ $\mathrm{MgCl}_{2}, 0.0067 \%$ Triton X-100), $10 \mu \mathrm{l} 25 \mathrm{mg} \mathrm{ml}^{-1}$ acetylated BSA, $5 \mu \mathrm{l} 10 \mu \mathrm{g} \mathrm{ml}^{-1}$ PNA probe). Sections were covered with a glass coverslip, denatured at $83^{\circ} \mathrm{C}$ for 4 min on a heating block and incubated in the dark in a humidified chamber overnight at room temperature. Slides were then washed, blocked and subsequently incubated with Alexa Fluor 488-conjugated donkey anti-goat antibodies (Invitrogen, A-11055, $4 \mu \mathrm{g} \mathrm{ml}^{-1}$ ), stained with DAPI and mounted in ProLong Gold antifade reagent (Invitrogen, P36934). Confocal images were obtained with a Nikon Ti-U inverted microscope with CSU-10 spinning disk confocal head (Spectral Boralis) using a Nikon Plan APO60x/1.4 lens and Hamamatsu Orca-ER camera. The Cy3 laser was held at a constant intensity to capture all images. Images from mice treated with lithium and Rspol were obtained in 16-bit grey scale and 8-bit RGB24 formats, respectively. To measure telomere lengths, quantitative image analysis was performed on confocal images using Media Cybernetics and ImageProPlus 7.01 software. The DAPI images were used to define the nuclear area in which to measure telomere intensities. Images were first deconvoluted, and nuclear outlines of interest were drawn and applied as a mask onto the $\mathrm{Cy} 3$ images. $\mathrm{Cy} 3$ images were also deconvoluted, and telomere intensities within each nucleus were obtained (for 16-bit images applied filters were: 1,000-65,535 pixel intensity threshold and area larger than 11.25sq. microns; for 8-bit images applied filters were: density/intensity ratio threshold of 0-254). Statistical analyses of differences in telomere lengths between samples were performed using the Mann-Whitney $U$-test. 
Isolation of intestinal crypt and stromal cells. Ileum of $8 \mathrm{~cm}$ was longitudinally cut and rinsed in cold PBS, followed by firm scraping of intestinal villi with haemacytometer coverslip, and incubation in $10 \mathrm{ml} 30 \mathrm{mM}$ EDTA and $1.5 \mathrm{mM}$ DTT in PBS on ice for $20 \mathrm{~min}$. The intestine was transferred to $10 \mathrm{ml} 30 \mathrm{mM}$ EDTA in PBS at $37^{\circ} \mathrm{C}$ for $8 \mathrm{~min}$, followed by 3 rounds of shaking per second for $30-40 \mathrm{~s}$. The supernatant was centrifuged at $200 \mathrm{~g}$ for $1 \mathrm{~min}$ at $4^{\circ} \mathrm{C}$, followed by passage through a $70 \mu \mathrm{m}$ cell strainer. The strained supernatant was spun down again at $130 \mathrm{~g}$ for $1 \mathrm{~min}$ at $4{ }^{\circ} \mathrm{C}$ to deplete single cells, and the crypt pellet was collected for RNA extraction. The remaining intestinal tissue is scraped with a coverslip to remove residual epithelial cells and is mechanically homogenized with a TissueRuptor (Qiagen) before RNA extraction.

RNA extraction. All RNA extractions were performed with the miRNeasy Kit (Qiagen). RNA quality was confirmed with Nanodrop spectrophotometry or Agilent Bioanalyzer.

Quantitative RT-PCR. Reverse transcription was performed with miScript II RT Kit (Qiagen) according to the manufacturer, using miScript HiFlex buffer. Realtime PCR was performed on a Roche LightCycler 480 using SYBR Green JumpStart Taq ReadyMix (Sigma). Reactions $(10 \mathrm{ml})$ were performed in triplicate, as follows: $10 \mathrm{~min}$ at $95^{\circ} \mathrm{C}, 45 \mathrm{cycles}$ of $15 \mathrm{~s}$ at $95^{\circ} \mathrm{C}, 30 \mathrm{~s}$ at $59^{\circ} \mathrm{C}, 30 \mathrm{~s}$ at $72^{\circ} \mathrm{C}$. Melt curve and gel electrophoretic analyses were performed to verify specific target amplification. Amplification from experimental samples was normalized to standard curves that were made from dilutions of pooled samples. Cp values from each amplification curve were computed by the second-derivative method, the messenger RNA expression levels were normalized to that of $\beta$-actin, and the mature miRNA expression levels were normalized to that of RNU6. Primer sequences are provided in Supplementary Table 4. Student's $t$-tests were used for comparisons, except that a two-way analysis of variance (multiple comparisons) was used for statistical analyses of human fibroblasts, comparing the gene expression at each dose of CHIR99021 to no drug within each genotype.

Microarray protocol and analysis. Microarray experiments were conducted by the University of Pennsylvania Molecular Profiling Facility, including quality control tests of the total RNA samples by Agilent Bioanalyzer and Nanodrop spectrophotometry. Crypt and stromal RNA was obtained, respectively, from three or two WT and three or four G4 $m T R^{-/-}$mice, with samples from each mouse analysed on an individual array. All protocols were conducted as described in the Ambion WT Expression Manual and the Affymetrix GeneChip Expression Analysis Technical Manual, using $250 \mathrm{ng}$ of total RNA for first-strand cDNA synthesis. cDNA yields ranged from 9.4 to $10.7 \mu \mathrm{g}$, and $5.5 \mu \mathrm{g}$ of labelled cDNA was hybridized to Mouse Gene 1.0ST GeneChips, stained with streptavidin-phycoerythrin, and visualized with a GeneChip 3000 7G scanner. Affymetrix probe intensity (.cel) files were imported into Partek Genomics Suite (v6.6, Partek Inc., St Louis, MO, USA) where RMA normalization was applied. The resulting $\log _{2}$-transformed intensities were filtered to exclude the IDs corresponding to technical controls, and analysed for differential expression using SAM (Significance Analysis of Microarrays, samr v2.0, Stanford University ${ }^{48}$ ), generating $q$-values (False Discovery Rate) and fold change for each Transcript ID. For the miRNA microarray, crypt RNA obtained from three G4 $m T R^{-1-}$ mice (same as that used in messenger RNA microarray), with samples from each mouse analysed on an individual array. Amplification steps were performed with the Genisphere FlashTag (miRNA) kit and the Affymetrix GeneChip miRNA 3.0 Array chips were used. Affymetrix probe intensity (.cel) files were imported into Partek Genomics Suite (v6.6, Partek Inc., St. Louis, MO, USA) where RMA normalization was applied. The resulting $\log _{2^{-}}$ transformed intensities were filtered to exclude the IDs corresponding to technical controls, and analysed for differential expression using SAM, generating $q$-values (False Discovery Rate) and fold change for each Transcript ID.

Heat map and gene set enrichment analysis (GSEA). Heat maps were generated with Multiple Experiment Viewer (MeV; ref. 49) v4.8.1 from the Dana-Farber Cancer Institute. GSEA (ref. 50) v2.0.13 from the Broad Institute was used to identify significant enrichments in the microarray data. The C2 collection containing 4850 curated gene sets from the Molecular Signature Database (MSigDB) v3.1 was used to identify enriched pathways in WT versus $\mathrm{G}_{4} \mathrm{mTR}^{-/-}$ crypts. Chip2Chip was used to translate gene identifiers from the $\mathrm{C} 2$ collection to the Affymetrix Mouse Gene 1.0ST probeset IDs. GSEA was performed as follows: $\log _{2}$-transformed RMA values from each individual sample were used for the expression data set. Parameter details are as follows: Permutations: 1,000, 'Collapse data set' $=$ FALSE, Permutation type $=$ gene_set, Max size $=5,000$, Min size $=1$. GSEA was also performed using user-defined gene sets from the transcriptomic profile of ISCs or downregulated genes in Lgr $4 / 5 \mathrm{KO}$ crypts, described as follows. 361 genes out of 379 genes defined as the transcriptomic profile of Lgr5 + ISCs on the Affymetrix platform ${ }^{51}$ matched the Affymetrix Mouse Gene 1.0ST platform that was used for measurements, and these were used to generate the heat map and GSEA enrichment plot. We also used the 125 genes out of 135 genes defined as the ISC transcriptome ${ }^{18}$ that matched our microarray platform. For the gene set describing downregulated genes in Lgr4/5 KO crypts $^{26}, 259$ out of 306 genes from the array platforms matched the Affymetrix platform and were used in GSEA.
GSEA was performed for proliferating (PD32) and replicatively senescent (PD88) IMR90 cells using publicly available Gene Expression Omnibus (GEO) data set GSE36640 (ref. 52). Chip2Chip was used to translate gene identifiers from the C2 collection to the Affymetrix Human Genome U133 Plus 2.0 Array. GSEA was performed as follows: raw data set values from each individual sample were used for the expression data set. Parameter details are as follows: Permutations: 1,000, 'Collapse data set' $=$ FALSE, Permutation type $=$ gene_set, Max size $=10,000$, Min size $=5$, and 4721 out of $4722 \mathrm{C} 2$ collection of gene sets from MsigDB v4.0 passed the criteria for analysis.

Crypt culture. Intestinal crypts used for culturing were isolated from the proximal half of the small intestine as described ${ }^{53}$. Briefly, villi were scraped off the small intestinal lumen with a glass slide, and the remaining tissue was cut in $2-4 \mathrm{~mm}$ pieces, incubated with $30 \mathrm{ml}$ of $30 \mathrm{mM}$ EDTA in PBS for $15 \mathrm{~min}$ on ice, with occasional inversion. The tissues were allowed to settle by gravity and subsequently, the mixture was washed with ice cold PBS, and the supernatant was discarded. The wash/discard cycle was repeated for 10-15 rounds or until the supernatant was composed predominantly of crypts, which was filtered through a $70 \mu \mathrm{m}$ cell strainer into a $50 \mathrm{ml}$ centrifuge tube and pelleted via centrifugation at $300 \mathrm{~g}$ for $5 \mathrm{~min}$. Isolated crypts were cultured in Matrigel (BD Biosciences) with advanced DMEM/F12 medium containing final and basal concentrations of $100 \mathrm{ng} \mathrm{ml}^{-1}$ noggin, $50 \mathrm{ng} \mathrm{ml}^{-1} \mathrm{mEGF}$ and $1 \mu \mathrm{g} \mathrm{ml}^{-1}$ RSpol (higher levels of RSpol were used for some experiments as indicated). Approximately 200-500 crypts per $50 \mu \mathrm{l}$ of Matrigel were plated per well in a pre-warmed 24-well plate and cultured at $37^{\circ} \mathrm{C}$ in a $6 \% \mathrm{CO}_{2}$ incubator with ambient $\mathrm{O}_{2}$. CHIR99021 (Tocris Biosciences) was prepared as a $2 \mathrm{mM}$ stock solution in DMSO. Lentiviruses were made at the Wistar Vector Core. Anti-miRNA plasmids targeting miR34a (MZIP34a-PA-1) and control plasmids (MZIP000-PA-1) were obtained from System Biosciences. Organoids were cultured and established in $5 \mu \mathrm{M}$ CHIR conditions for several passages before infection. Organoids embedded in Matrigel were counted and washed once with cold PBS, and the organoids and Matrigel were broken up by pipetting. Organoids were spun down at $300 \mathrm{~g}$ in a cold centrifuge and resuspended in culture media containing $5 \mu \mathrm{M}$ CHIR. Lentiviruses were added to the organoid suspension at different multiplicities of infection (MOI of 25 or 50) for $4-6 \mathrm{~h}$ in a $37^{\circ} \mathrm{C}$ incubator. After incubation, organoids were collected and spun down at $300 \mathrm{~g}$, resuspended in Matrigel, plated, and grown at $5 \mu \mathrm{M}$ CHIR for $2-3$ days before $2 \mu \mathrm{g} \mathrm{ml}^{-1}$ puromycin selection.

Cell lines. Primary human fibroblasts were obtained from the Coriell Institute The two DC lines were: GM01774 (population doubling level (PD) 25; from 7-year-old male), and AG04646 (PD 21; from 11-year-old male). The three healthy control (WT) lines were: GM01786 (PD 25; 30-year-old mother of GM01774), GM00409 (PD 16; from 7-year-old male) and GM00323 (PD 23.6; from 11-yearold male). The Werner syndrome line was AG05229B (PD 30; from 25-year-old male), which was retrovirally transduced with pBABE-puro-hTERT, or with the empty pBABE-puro vector as a control. WT, $m i R 34 a^{-/-}$, sibling-matched G4 $m T R^{-1-} m i R 34 a^{+/+}, \mathrm{G} 4 m T R^{-1-} m i R 34 a^{-1-}$ mouse skin fibroblasts were cultured from ear clippings. Mycoplasma contamination testing was not performed on these cultures, but general testing of cell cultures in our laboratory has not revealed mycoplasma contamination. miR34a overexpression and control retroviral vectors (pMSCV-PIG-34a and pMSCV-PIG) were obtained from Joshua Mendell. miR34a $a^{-1}$ fibroblasts were transduced with the retroviruses and selected with puromycin. Fibroblasts were cultured in DMEM with 15\% FBS, with 1X penicillin/ streptomycin/amphotericin- $\mathrm{B}$ at $37^{\circ} \mathrm{C}$ in a $6 \% \mathrm{CO}_{2}$ and $3 \% \mathrm{O}_{2}$ atmosphere.

miR34a target prediction and 3'UTR reporter assays. Several databases were used to predict miR34a target sites on candidate genes. Candidate genes are Wnt pathway genes that have not yet been experimentally tested as miR34a targets elsewhere in literature (Supplementary Table 3). Candidate genes were screened with microRNA.org (http://www.microrna.org/), mirdb.org (http://mirdb.org/ miRDB/), TargetScan (http://www.targetscan.org/) and RNA22 (https://cm.jefferson.edu/rna22/Precomputed) to identify potential miR34a target sequences. The $3^{\prime}$ UTR of Lgr4 (NM_172671; + 1 to +1728 ) was PCR amplified from genomic mouse tail DNA and subcloned into the XhoI and NotI sites downstream of renilla luciferase of the psiCHECK2 vector (Promega). Mouse skin fibroblasts were transfected with $500 \mathrm{ng}$ of reporter construct with Fugene 6 transfection reagent (Promega). Cells were lysed $24 \mathrm{~h}$ after transfection and the luciferase activity was measured by the Dual-GLO luciferase assay (Promega).

Statistics. Bar graphs are represented with s.e.m. error bars unless otherwise stated. $P$ values were calculated with Prism. Unpaired $t$-tests assuming equal population s.d. were performed unless specified. Two-way analysis of variance was performed for Supplementary Fig. 14.

Data availability. The gene expression profiling microarray data generated during the study have been deposited in Gene Expression Omnibus (GEO) with the accession code GSE90820 (https://www.ncbi.nlm.nih.gov/geo/query/ acc.cgi?acc=GSE90820). The remaining data that support the findings of this study are available from the authors upon request. 


\section{References}

1. Barker, N. Adult intestinal stem cells: critical drivers of epithelial homeostasis and regeneration. Nat. Rev. Mol. Cell Biol. 15, 19-33 (2014).

2. Clevers, H., Loh, K. M. \& Nusse, R. Stem cell signaling. An integral program for tissue renewal and regeneration: Wnt signaling and stem cell control. Science 346, 1248012 (2014).

3. Yan, K. S. et al. The intestinal stem cell markers Bmil and Lgr5 identify two functionally distinct populations. Proc. Natl Acad. Sci. USA 109, 466-471 (2012)

4. Sangiorgi, E. \& Capecchi, M. R. Bmil is expressed in vivo in intestinal stem cells. Nat. Genet. 40, 915-920 (2008).

5. Tian, H. et al. A reserve stem cell population in small intestine renders Lgr5positive cells dispensable. Nature 478, 255-259 (2011).

6. Li, N. et al. Single-cell analysis of proxy reporter allele-marked epithelial cells establishes intestinal stem cell hierarchy. Stem Cell Rep. 3, 876-891 (2014)

7. Sandin, S. \& Rhodes, D. Telomerase structure. Curr. Opin. Struct. Biol. 25, 104-110 (2014).

8. Armanios, M. Telomeres and age-related disease: how telomere biology informs clinical paradigms. J. Clin. Invest. 123, 996-1002 (2013).

9. Blasco, M. A. et al. Telomere shortening and tumor formation by mouse cells lacking telomerase RNA. Cell 91, 25-34 (1997).

10. Rudolph, K. L. et al. Longevity, stress response, and cancer in aging telomerasedeficient mice. Cell 96, 701-712 (1999).

11. Lee, H. W. et al. Essential role of mouse telomerase in highly proliferative organs. Nature 392, 569-574 (1998).

12. Flores, I., Cayuela, M. L. \& Blasco, M. A. Effects of telomerase and telomere length on epidermal stem cell behavior. Science 309, 1253-1256 (2005).

13. Begus-Nahrmann, Y. et al. p53 deletion impairs clearance of chromosomalinstable stem cells in aging telomere-dysfunctional mice. Nat. Genet. 41, 1138-1143 (2009).

14. Tao, S. et al. Wnt activity and basal niche position sensitize intestinal stem and progenitor cells to DNA damage. EMBO J. 34, 624-640 (2015).

15. Park, J. I. et al. Telomerase modulates Wnt signalling by association with target gene chromatin. Nature 460, 66-72 (2009).

16. Hoffmeyer, K. et al. Wnt/beta-catenin signaling regulates telomerase in stem cells and cancer cells. Science 336, 1549-1554 (2012).

17. Zhang, Y., Toh, L., Lau, P. \& Wang, X. Human telomerase reverse transcriptase (hTERT) is a novel target of the Wnt/beta-catenin pathway in human cancer. J. Biol. Chem. 287, 32494-32511 (2012).

18. van der Flier, L. G. et al. Transcription factor achaete scute-like 2 controls intestinal stem cell fate. Cell 136, 903-912 (2009).

19. Martinez, P. \& Blasco, M. A. Telomeric and extra-telomeric roles for telomerase and the telomere-binding proteins. Nat. Rev. Cancer 11, 161-176 (2011).

20. Farin, H. F., Van Es, J. H. \& Clevers, H. Redundant sources of Wnt regulate intestinal stem cells and promote formation of Paneth cells. Gastroenterology 143, 1518-1529 (2012).

21. Sato, T. et al. Paneth cells constitute the niche for Lgr5 stem cells in intestinal crypts. Nature 469, 415-418 (2011).

22. Strong, M. A. et al. Phenotypes in $\mathrm{mTERT}^{+/-}$and $\mathrm{mTERT}^{+/-}$mice are due to short telomeres, not telomere-independent functions of telomerase reverse transcriptase. Mol. Cell Biol. 31, 2369-2379 (2011).

23. Listerman, I., Gazzaniga, F. S. \& Blackburn, E. H. An investigation of the effects of the core protein telomerase reverse transcriptase on Wnt signaling in breast cancer cells. Mol. Cell Biol. 34, 280-289 (2014).

24. Kim, N. H. et al. p53 and microRNA-34 are suppressors of canonical Wnt signaling. Sci. Signal 4, ra71 (2011).

25. Herbig, U., Jobling, W. A., Chen, B. P., Chen, D. J. \& Sedivy, J. M. Telomere shortening triggers senescence of human cells through a pathway involving ATM, p53, and p21(CIP1), but not p16(INK4a). Mol. Cell 14, 501-513 (2004)

26. de Lau, W. et al. Lgr5 homologues associate with Wnt receptors and mediate R-spondin signalling. Nature 476, 293-297 (2011).

27. Carmon, K. S., Lin, Q., Gong, X., Thomas, A. \& Liu, Q. LGR5 interacts and cointernalizes with Wnt receptors to modulate Wnt/beta-catenin signaling. Mol. Cell Biol. 32, 2054-2064 (2012).

28. Klein, P. S. \& Melton, D. A. A molecular mechanism for the effect of lithium on development. Proc. Natl Acad. Sci. USA 93, 8455-8459 (1996).

29. Yin, X. et al. Niche-independent high-purity cultures of Lgr5 ${ }^{+}$intestinal stem cells and their progeny. Nat. Methods 11, 106-112 (2014).

30. Palm, W. \& de Lange, T. How shelterin protects mammalian telomeres. Annu. Rev. Genet. 42, 301-334 (2008).

31. Diala, I. et al. Telomere protection and TRF2 expression are enhanced by the canonical Wnt signalling pathway. EMBO Rep. 14, 356-363 (2013).

32. Karlseder, J., Smogorzewska, A. \& de Lange, T. Senescence induced by altered telomere state, not telomere loss. Science 295, 2446-2449 (2002).
33. Sansom, O. J. et al. Loss of Apc in vivo immediately perturbs Wnt signaling, differentiation, and migration. Genes Dev. 18, 1385-1390 (2004).

34. Fevr, T., Robine, S., Louvard, D. \& Huelsken, J. Wnt/beta-catenin is essential for intestinal homeostasis and maintenance of intestinal stem cells. Mol. Cell Biol. 27, 7551-7559 (2007).

35. Zhou, W. J., Geng, Z. H., Spence, J. R. \& Geng, J. G. Induction of intestinal stem cells by R-spondin 1 and Slit2 augments chemoradioprotection. Nature 501, 107-111 (2013).

36. Wang, X. et al. Pharmacologically blocking p53-dependent apoptosis protects intestinal stem cells and mice from radiation. Sci. Rep. 5, 8566 (2015).

37. O'Sullivan, J. N. et al. Chromosomal instability in ulcerative colitis is related to telomere shortening. Nat. Genet. 32, 280-284 (2002).

38. Risques, R. A. et al. Ulcerative colitis is a disease of accelerated colon aging: evidence from telomere attrition and DNA damage. Gastroenterology 135, 410-418 (2008).

39. Jonassaint, N. L., Guo, N., Califano, J. A., Montgomery, E. A. \& Armanios, M. The gastrointestinal manifestations of telomere-mediated disease. Aging Cell 12, 319-323 (2013)

40. Florian, M. C. et al. A canonical to non-canonical Wnt signalling switch in haematopoietic stem-cell ageing. Nature 503, 392-396 (2013).

41. Reya, T. et al. A role for Wnt signalling in self-renewal of haematopoietic stem cells. Nature 423, 409-414 (2003).

42. Famili, F. et al. Discrete roles of canonical and non-canonical Wnt signaling in hematopoiesis and lymphopoiesis. Cell Death Dis. 6, e1981 (2015).

43. Martinsson, L. et al. Long-term lithium treatment in bipolar disorder is associated with longer leukocyte telomeres. Transl. Psychiatry 3, e261 (2013).

44. Squassina, A. et al. Leukocyte telomere length positively correlates with duration of lithium treatment in bipolar disorder patients. Eur. Neuropsychopharmacol. 26, 1241-1247 (2016).

45. Pottegard, A. et al. Long-term use of lithium and risk of colorectal adenocarcinoma: a nationwide case-control study. Br. J. Cancer 114, 571-575 (2016).

46. Wei, Q. et al. R-spondin1 is a high affinity ligand for LRP6 and induces LRP6 phosphorylation and beta-catenin signaling. J. Biol. Chem. 282, 15903-15911 (2007).

47. Suram, A. et al. Oncogene-induced telomere dysfunction enforces cellular senescence in human cancer precursor lesions. EMBO J. 31, 2839-2851 (2012).

48. Tusher, V. G., Tibshirani, R. \& Chu, G. Significance analysis of microarrays applied to the ionizing radiation response. Proc. Natl Acad. Sci. USA 98, 5116-5121 (2001).

49. Saeed, A. I. et al. TM4: a free, open-source system for microarray data management and analysis. Biotechniques 34, 374-378 (2003).

50. Subramanian, A. et al. Gene set enrichment analysis: a knowledge-based approach for interpreting genome-wide expression profiles. Proc. Natl Acad. Sci. USA 102, 15545-15550 (2005).

51. Munoz, J. et al. The Lgr5 intestinal stem cell signature: robust expression of proposed quiescent ' +4 ' cell markers. EMBO J. 31, 3079-3091 (2012).

52. Shah, P. P. et al. Lamin B1 depletion in senescent cells triggers large-scale changes in gene expression and the chromatin landscape. Genes Dev. 27, 1787-1799 (2013).

53. Sato, T. \& Clevers, H. Primary mouse small intestinal epithelial cell cultures. Methods Mol. Biol. 945, 319-328 (2013).

\section{Acknowledgements}

We thank members of the Johnson, Lynch, Rustgi and Lengner laboratories and P. Adams for helpful discussions, R. Pignolo and Y. Zhang for collaborative efforts that helped lead to our studies, and J. Huang and P. Klein for GSK inhibitor advice. This work was supported P01 AG031862, R21 AG054209 and U01 DK007066, a pilot grant from the University of Pennsylvania Institute on Aging, and the Penn Center for Molecular Studies in Digestive and Liver Diseases (P30DK050306) and its core facilities. T.-L.B.Y. was supported by a Canadian Institutes of Health Research Doctoral Research Award.

\section{Author contributions}

T.-L.B.Y., Q.C. and F.B.J. designed the experiments, interpreted data and wrote the manuscript with contributions from C.J.L., J.P.L., A.K.R., J.W.T. and the other authors. Q.C. and T.-L.B.Y. carried out experiments with assistance from the other authors.

\section{Additional information}

Supplementary Information accompanies this paper at http://www.nature.com/ naturecommunications

Competing financial interests: The authors declare no competing financial interests. 
Reprints and permission information is available online at http://npg.nature.com/ reprintsandpermissions/

How to cite this article: Yang, T.-L. B. et al. Mutual reinforcement between telomere capping and canonical Wnt signalling in the intestinal stem cell niche. Nat. Commun. 8, 14766 doi: 10.1038/ncomms14766 (2017).

Publisher's note: Springer Nature remains neutral with regard to jurisdictional claims in published maps and institutional affiliations. (c) (i) This work is licensed under a Creative Commons Attribution 4.0 International License. The images or other third party material in this article are included in the article's Creative Commons license, unless indicated otherwise in the credit line; if the material is not included under the Creative Commons license, users will need to obtain permission from the license holder to reproduce the material. To view a copy of this license, visit http://creativecommons.org/licenses/by/4.0/

(C) The Author(s) 2017 